\title{
A new facet of NDRG1 in pancreatic ductal adenocarcinoma: Suppression of glycolytic metabolism
}

\author{
WENSHENG LIU ${ }^{1-3^{*}}$, BO ZHANG ${ }^{1-3^{*}}$, QIANGSHENG HU ${ }^{1-3^{*}}$, YI QIN $^{1-3}$, WENYAN XU $^{1-3}$, \\ SI SHI ${ }^{1-3}$, CHEN LIANG ${ }^{1-3}$, QINGCAI MENG ${ }^{1-3}$, JINFENG XIANG ${ }^{1-3}$, DINGKONG LIANG ${ }^{1-3}$, \\ SHUNRONG JI ${ }^{1-3}$, JIANG LIU ${ }^{1-3}$, PENGFEI HU ${ }^{3}$, LIANG LIU ${ }^{1-3}$, CHEN LIU ${ }^{1-3}$, \\ JIANG LONG ${ }^{1-3}$, QUANXING NI ${ }^{1-3}$, XIANJUN YU ${ }^{1-3}$ and JIN XU ${ }^{1-3}$ \\ ${ }^{1}$ Department of Pancreatic Surgery, Fudan University Shanghai Cancer Center, Shanghai; \\ ${ }^{2}$ Department of Oncology, Shanghai Medical College, Fudan University, Shanghai; \\ ${ }^{3}$ Pancreatic Cancer Institute, Fudan University, Shanghai, P.R. China
}

Received December 20, 2016; Accepted March 7, 2017

DOI: 10.3892/ijo.2017.3938

\begin{abstract}
N-myc downstream-regulated gene 1 (NDRG1) is known as tumor/metastasis suppressor in a variety of cancers including pancreas, being involved in angiogenesis, cancer growth and metastasis. However, the precise molecular mechanism how NDRG1 exerts its inhibitory function in pancreatic cancer remains unclear. In this investigation, we demonstrated that K-Ras plays a vital role in modulating NDRG1 protein level in PDAC cancer cells in vitro, which is mediated through ERK signaling. Noteworthy, $\mathrm{K}$-Ras downstream Akt/mTOR signaling is inhibited upon NDRG1 overexpression, resulting in decease of HIF1 $\alpha$ level. Moreover, NDRG1 has a unique role in modulating cancer metabolism of pancreatic ductal adenocarcinoma (PDAC). The mechanism accounting for NDRG1 in modulating aerobic glycolysis, at least partly, relied on its regulation of glycolysis genes including GLUT1, HK2, LDHA and PDK1. Additionally, NDRG1 is shown to suppress the activity of HIF1 $\alpha$, which is responsible for regulation of glycolysis enzymes. The current study is the first to elucidate a unique facet of the potent tumor/metastasis suppressor NDRG1 in the regulation of PDAC glycolysis, leading to important insights into the mechanism by which NDRG1 exert inhibitory function in PDAC.
\end{abstract}

Correspondence to: Professor Xianjun Yu or Dr Jin Xu, Pancreatic Cancer Institute, Fudan University, 270 Dong An Road, Shanghai 200032, P.R. China

E-mail: xujin@fudanpci.org

E-mail: yuxianjun@fudanpci.org

${ }^{*}$ Contributed equally

Key words: N-myc downstream-regulated gene 1, pancreatic ductal adenocarcinoma, cancer metabolism, glycolysis, hypoxia-inducible factor $1 \alpha$

\section{Introduction}

As one of the most lethal and aggressive cancers, pancreatic ductal adenocarcinoma (PDAC) is associated with a very poor prognosis (1). As such, it remains a clinically challenging disease to treat with a 5-year survival rate of 5\%, despite that continual improvements have been made in therapies in recent decades (1). PDAC has several defining features that influence its aggressive biology and resistance to multiple therapeutic modalities, which is characterized by a distinct and exuberant stromal reaction (desmoplasia), together with the poor vascularization, creating a highly hypoxic and nutrient-poor microenvironment, accompanied with genomic complexity and an altered metabolism $(2,3)$. Moreover, the aforementioned tumor biology of PDAC contributes to uncontrolled cancer growth, early metastasis and recurrence, resistance to chemotherapy and radiotherapy, and finally short survival $(1,3)$.

Metabolic alterations have been regarded as a hallmark of cancer cells, which is an important research area that has gained increasing attention in recent years (4). Unlike majority of normal cells which depend on mitochondrial oxidative phosphorylation to provide energy, cancer cells apply aerobic glycolysis as their primary energy resource (4). Several molecules, including Oncogenic K-Ras, hypoxia-inducible factor $1 \alpha$ and c-Myc, have been suggested to play important roles in promoting glycolysis and thus be involved in 'metabolic reprogramming' in the cancer cells (5-8). Oncogenic K-Ras mutation, which occurs in typical cases, is the signature event in PDAC, serving a critical role in tumor initiation (5). There are considerable evidences showing that K-Ras also plays vital roles in metabolic changes in PDAC cancer cells and promotes cancer development by inducing mitochondrial dysfunction (5). The HIFl $\alpha$ pathway enables tumor cells to survive by changing glucose metabolism toward a glycolytic phenotype, regulating $\mathrm{pH}$ balance and proliferation rate, and eventually angiogenesis (6). c-Myc induces aerobic glycolysis by enhancing glucose uptake and lactate production as well as providing glycolytic intermediates for nucleotide, amino acid, and lipid biosynthesis $(7,8)$. In this regard, an understanding of cellular metabolic derangements in PDAC could lead to novel therapeutic approaches. 
$\mathrm{N}$-myc downstream regulated gene 1 (NDRG1) (also known as Drg1, RTP, Rit42, PROXY-1 or Cap43), a member of the NDRG family, has been well described as a tumor/metastasis suppressor in a number of cancers (9), including pancreas (10). It is believed that NDRG1 participates in suppression of tumourigenesis or carcinogenesis (10). However, it is unclear whether NDRG1 is involved in cancer metabolism changes, which is a critical aspect during carcinogenesis and metastasis. Previously, we demonstrated NDRG1 exerts inhibition of colonic and prostate cancer cell migration by blocking Src activation (11). Of note, studies revealed that Src regulate breast cancer cell glucose metabolism by reducing c-Myc translation and transcription of the glucose transporter GLUT1 (12). Therefore, it is reasonable to speculate NDRG1 may play a role in cancer cell metabolism regulation. In this study, we examined whether NDRG1 expression could play any role in PDAC cancer cell metabolism. Moreover, we also investigated the potential target molecules of NDRG1 involved in the regulation of PDAC cancer cell metabolism.

\section{Materials and methods}

Cells and reagents. The human pancreatic cancer cell lines MIA PaCa-2 and PANC-1 cells were obtained from ATCC and cultured in DMEM supplemented with 10\% FBS. All of the cell culture media contained $100 \mathrm{U} / \mathrm{ml}$ penicillin and $100 \mathrm{mg} / \mathrm{ml}$ streptomycin. iKras cell lines expressing K-Ras ${ }^{\mathrm{G} 12 \mathrm{D}}$ upon doxycycline (Dox) treatment was generously provided by Professor Paul J. Chiao from MD Anderson Cancer Center. Doxycycline and MAPK inhibitor U0126 were obtained from Selleck and used at a final concentration of $2 \mu \mathrm{g} / \mathrm{ml}$ and $10 \mu \mathrm{M}$, respectively.

Plasmid and stable cell line generation. In order to overexpress NDRG1 in MIA PaCa-2 and PANC-1 cells, we generated NDRG1 overexpression constructs. pCDH-CMV-MCS-EF1Puro (System Biosciences) plamid was used to generate NDRG1 expression constructs. NDRG1 lentiviral expression construct was co-transfected with psPAX2 and pMD2.G into HEK293T cells to produce lentiviral particles. Stable cell lines were obtained by infection with lentiviral particles and followed by puromycin selection.

siRNA transfection. In order to silence ERK1 expression in pancreatic cancer cells, we silenced ERK1 expression by using siRNA mediated silencing. siRNA targets against ERK1 were CCUGCUGGACCGGAUGUUA and UCAUGGAGA CAGACCUGTA, respectively.

Cell proliferation assay and colony formation assay. Cell proliferation was examined using water-soluble tetrazolium salt assay using the Cell Counting Kit-8. In short, cells $\left(1.5 \times 10^{3} /\right.$ well $)$ were seeded in 96 -well culture plates in triplicate and incubated for 4 days at $37^{\circ} \mathrm{C} / 5 \% \mathrm{CO}_{2}$ in a humidified incubator. Viable cells were quantified at each $24 \mathrm{~h}$ interval by measuring $\mathrm{OD}_{450}$ using microplate reader.

Cells were seeded in triplicate in 6-well plates at an initial density of 500 cells/well. After 10-14 days, colonies were
Table I. Primers used in RT-PCR analysis.

\begin{tabular}{ll}
\hline Gene & \multicolumn{1}{c}{ Primer sequence } \\
\hline GLUT1 & 5'-CTTTGTGGCCTTCTTTGAAGT-3' \\
& 5'-CCACACAGTTGCTCCACAT-3' \\
HK2 & 5'-GATTGTCCGTAACATTCTCATCGA-3' \\
& 5'-TGTCTTGAGCCGCTCTGAGAT-3' \\
LDHA & 5'-TGGAGATTCCAGTGTGCCTGTATGG-3' \\
& 5'-CACCTCATAAGCACTCTCAACCACC-3' \\
PDK1 & 5'-CTGCACCAAGACCTCGTGTTGAGA-3' \\
& 5'-GGCAGCTTTGTTATACACTGGGAG-3' \\
3-actin & 5'-CTACGTCGCCCTGGACTTCGAGC-3' \\
& 5'-GATGGAGCCGCCGATCCACACGG-3' \\
\hline
\end{tabular}

The primers of GLUT1, HK2, LDHA, PDK1 and $\beta$-actin analyzed by RT-PCR.

clearly visible, and the cells were fixed with $4 \%$ paraformaldehyde for $15 \mathrm{~min}$ at room temperature and stained with $4 \mathrm{mg} / \mathrm{ml}$ of crystal violet. The colonies containing more than 50 cells were counted using light microscopy. The average number of colonies was determined from three independent experiments.

Western blotting. Western blotting was carried out as previously described (13). Briefly, whole-cell protein lysates were extracted, separated by SDS-PAGE and followed by immunoblotting. Antibodies used were purchased from designated manufacturers, NDRG1 (Abcam, ab133572), GLUT1 (Proteintech, 21829-1-AP), HK2 (Proteintech, 22029-1-AP), PDK1 (Proteintech, 100261-AP), LDHA (Proteintech, 19987-1-AP), HIF1 $\alpha$ (Proteintech, 20960-1-AP), K-Ras (Proteintech, 12063-1-AP), ERK1 (Abcam, ab119933), p44/p42 MAPK (ERK1/2) Rabbit mAb (Cell Signaling Technology, 4695), Phspho-p44/p42 MAPK(ERK1/2) (Thr202/Tyr204) (Cell Signaling Technology, 4370), Akt1 (Proteintech, 10176-2-AP), Anti-Akt1 (Phospho S473) (Abcam, ab81283), Anti-S6K (Abcam, ab32529), phosphor-p70S6 kinase (Thr389) (Cell Signaling Technology, 9234) and $\beta$-actin (Proteintech, 60008-1-Ig).

Oxygen consumption rate and extracellular acidification rate. Cellular mitochondrial function was measured using the Seahorse XF Cell Mito Stress test kit and the Bioscience XF96 Extracellular Flux Analyzer, according to the manufacturer's instructions. The glycolytic capacity was determined using the Glycolysis Stress test kit as per the manufacturer's instructions. Briefly, $4 \times 10^{4}$ cells were seeded onto 96-well plates and incubated overnight. After washing the cells with Seahorse buffer (DMEM with phenol red containing $25 \mathrm{mmol} / 1$ glucose, $2 \mathrm{mmol} / 1$ sodium pyruvate, and $2 \mathrm{mmol} / 1$ glutamine), $175 \mathrm{ml}$ of Seahorse buffer plus $25 \mathrm{ml}$ each of $1 \mathrm{mmol} / 1$ oligomycin, $1 \mathrm{mmol} / 1 \mathrm{FCCP}$, and $1 \mathrm{mmol} / 1$ rotenone were automatically injected to measure the oxygen consumption rate (OCR). Then, $25 \mathrm{ml}$ each of $10 \mathrm{mmol} / 1$ glucose, $1 \mathrm{mmol} / 1$ oligomycin, and $100 \mathrm{mmol} / 1$ 2-deoxy-glucose were added to measure the extracellular 
A

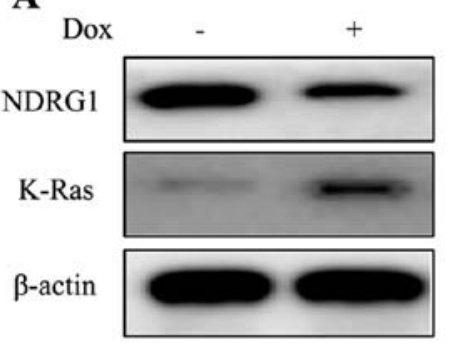

C

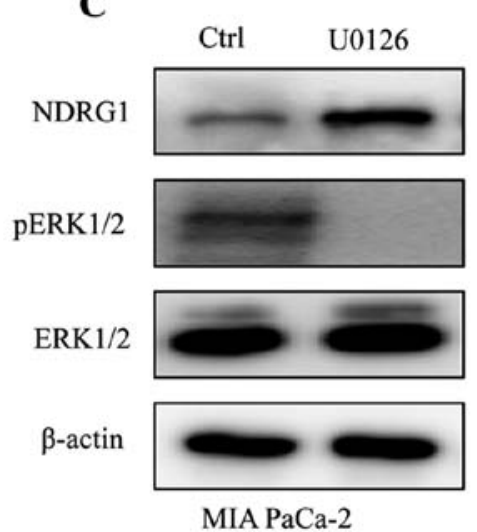

B

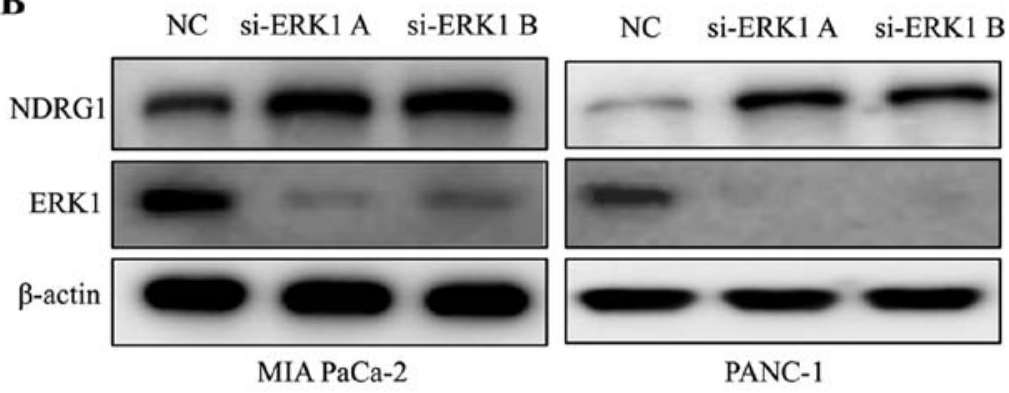

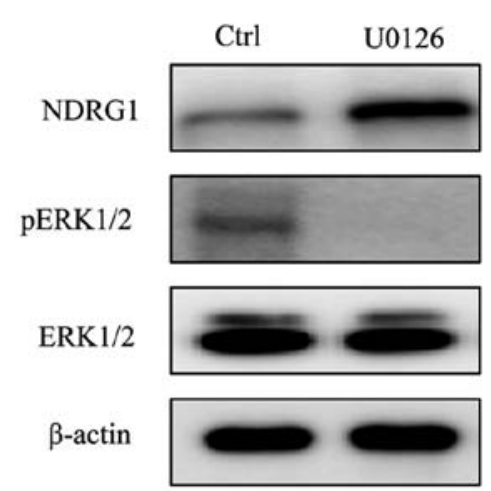

PANC-1

Figure 1. K-Ras downregulates NDRG1 expression via ERK1 in PDAC. (A) Whole-cell lysates were prepared, and immunoblotting was performed to determine the NDRG1 protein level by inducing oncogenic K-Ras expression in iKras cells. Blots are representative of 3-5 experiments. (B) ERK1 protein was mocked with specific siRNA, and NDRG1 protein level examination followed. (C) ERK activity was blocked by U0126, and NDRG1 protein level examination followed.

acidification rate (ECAR). The OCR and ECAR values were calculated after normalization to the cell number and are plotted as the mean \pm SD.

Quantitative real-time PCR. Total RNA was extracted using TRIzol reagent (Invitrogen). cDNA was synthesized by reverse transcription using a Takara PrimeScript RT reagent kit. The expression status of candidate genes and $\beta$-actin were determined by quantitative real-time PCR using an ABI 7900HT Real-time PCR system (Applied Biosystems). The reactions were run in triplicate. Primer sequences are listed in Table I.

Hypoxia response element (HRE) activity reporter assay. HRE activity was measured using a Dual-Glo Luciferase Assay System according to the manufacturer's protocol (Promega, E2920). HRE-Luciferase reported construct containing three hypoxia response elements from the Pgk-1 gene was obtained from Addgene (Addgene Plasmid 26731) (14).

Tissue specimens. The clinical tissue samples used in this study were obtained from patients diagnosed with pancreatic cancer at Fudan University Shanghai Cancer Center from 2010 to 2015. Prior patient consent and approval from the institutional research ethics committee were obtained.

Statistical analysis. Experiments were repeated at least three times. All data are presented as the mean \pm SD. Two-tailed unpaired Student's t-tests and one-way analysis of variance were used to evaluate the data. SPSS version 16.0 software
(IBM) was used for the data analysis. Differences were considered significant at $\mathrm{P}<0.05$.

\section{Results}

K-Ras downregulates NDRG1 expression via ERK1 in PDAC. Considering that K-Ras mutation is the signature of typical PDAC, we conducted experiments to elucidate the expression of NDRG1 in K-Ras network. By inducing oncogenic K-Ras expression in iKras cells, we observed that upon the presence of mutant K-Ras expression, the protein level of NDRG1 decreased significantly, indicating that NDRG1 might be a downstream effector of oncogenic K-Ras (Fig. 1A). It is well studied that oncogenic K-Ras mutation resulted in constitutive activation of MAPK, therefore, we examined the effect of MAPK on NDRG1 expression. We silenced ERK1 (MAPK3) expression in K-Ras mutated MIA PaCa-2 and PANC-1 cells, and observed that protein level of NDRG1 increased when ERK1 was silenced, indicating that NDRG1 may serve as a downstream target of Ras/MAPK signaling pathway (Fig. 1B). Next, we treated MIA PaCa-2 and PANC-1 cells with MAPK inhibitor UO126 and observed that NDRG1 protein level increased in UO126 treated cancer cells (Fig. 1C). Taken together, these results suggest that NDRG1 might be a downstream target of oncogenic K-Ras/MAPK axis.

Elevated NDRG1 expression suppresses proliferation of pancreatic cancer cells in vitro. To verify the suppressive function of NDRG1 in terms of inhibiting proliferation in pancreatic cancer, two pancreatic cancer cell lines, MIA 
A

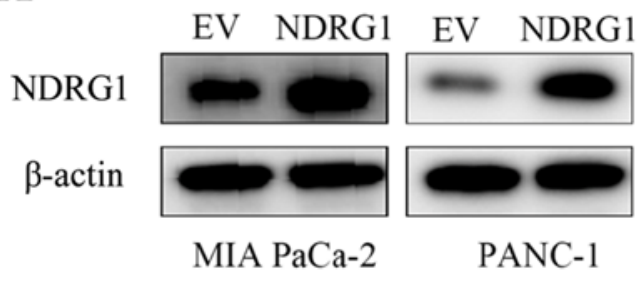

C

MIA PaCa-2

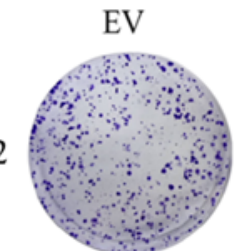

PANC-1
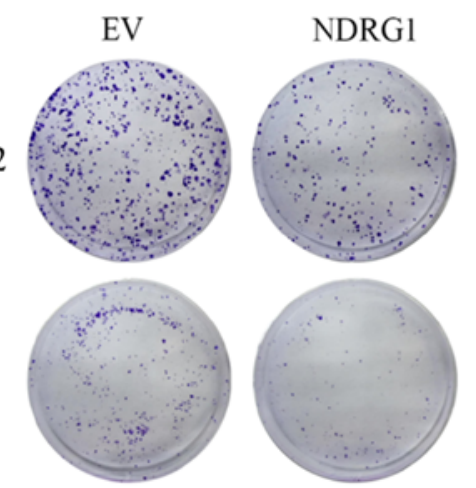

B

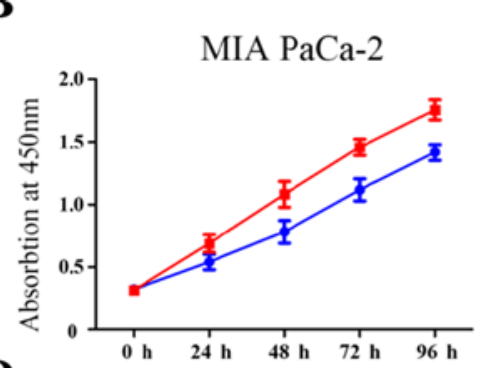

D

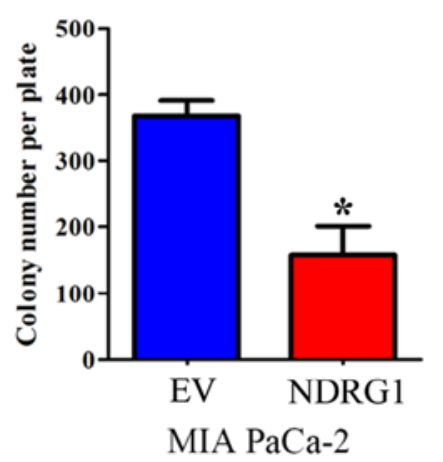

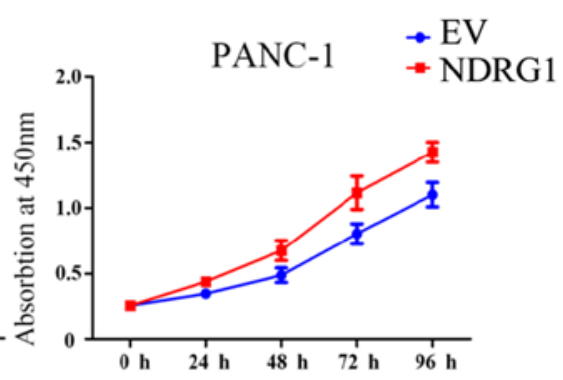

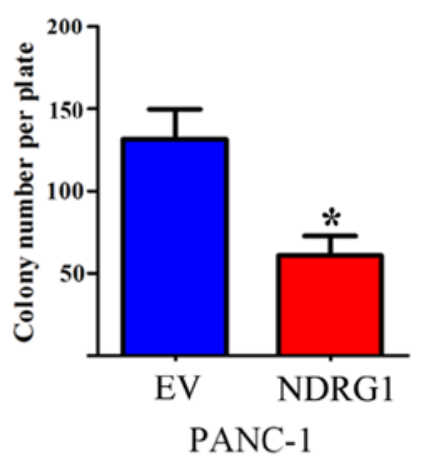

Figure 2. Elevated NDRG1 expression suppresses proliferation of pancreatic cancer cells in vitro. (A) Immunoblotting was conducted to examine NDRG1 expression in MIA PaCa-2 and PANC-1 cells transfected with NDRG1 overexpressing lentiviral particles. (B) CCK-8 assay was performed to determine cancer cell growth of the two pancreatic cancer lines. Data represent the means \pm SD from three independent experiments. (C and D) Colony formation assay was utilized to assess the effect of NDRG1 on colony formation ability of pancreatic cancer cells. Data represent the means \pm SD from three independent experiments. ${ }^{*} \mathrm{P}<0.05$, relative to control cells.

PaCa-2 and PANC-1, were utilized and infected with NDRG1 overexpressing lentiviral particles. The transfected MIA PaCa-2 and PANC-1 cells demonstrated a significant increase in NDRG1 expression relative to their empty-vector-infected control cells (Fig. 2A). CCK-8 assay was then performed to determine the growth repression role of the potent metastasis suppressor NDRG1. As indicated, the growth of MIA PaCa-2 was remarkably reduced with NDRG1 overexpression, relative to MIA PaCa-2 empty-vector control cells. Similar result was observed in the other pancreatic cancer cell line PANC-1 (Fig. 2B). To further assess the effects of NDRG1, the role of NDRG1 expression on cell proliferation was further investigated by a subsequent colony formation assay, in which MIA PaCa-2 and PANC-1 cancer cell colonies were markedly $(\mathrm{P}<0.05)$ decreased upon NDRG1 expression, relative to their empty-vector-transfected control cells (Fig. 2C and D). These data confirmed that NDRG1 inhibits proliferation and colony formation of pancreatic cancer cells in vitro, which is in good agreement with previous study (10).

NDRG1 decreases HIFl $\alpha$ expression through MAPK and Akt/ mTOR signaling. The ERK1 (MAPK3) and Akt/mammalian target of rapamycin (mTOR) signaling pathway are directly activated by Ras activation and could be the major mediators of Ras-driven oncogenesis (15). Regarding the interesting phenomenon that K-Ras has an inhibitory effect on NDRG1 protein in PDAC cancer cells (Fig. 1), while NDRG1 abrogate cancer cell growth of PDAC cancer cells (Fig. 2), we decided to decipher whether the K-Ras downstream signaling was changed upon NDRG1 overexpression. Herein, we performed western blotting to observe the phosphorylated and total protein level of Akt1, the direct mTOR target p70/p85 S6 kinase (S6K) and
ERK1. Noteworthy, in both cancer cell lines, elevated NDRG1 led to marked decrease of phosphorylated Akt1, S6K and ERK1 protein level relative to control cells, while there was no change on total Akt1, S6K and ERK1 protein level, indicating NDRG1 has inhibitory effect on MAPK and Akt/mTOR signaling (Fig. 3A).

$\mathrm{Akt} / \mathrm{mTOR}$ signaling has been shown to stimulate translation of HIF1 $\alpha$ mRNA and elevate HIF1 $\alpha$ protein level (16), which has been implicated in regulating many of the genes, contributing to 'Warburg effect' providing cancer cells with a growth advantage $(4,17)$. These results above implied that HIF1 $\alpha$ was a potential target of NDRG1 in terms of inhibiting cancer cell growth. Therefore, we conducted additional studies to investigate alterations on HIF1 $\alpha$ in the two pancreatic cancer cell lines and human PDAC specimens. Importantly, HIF1 $\alpha$ was reduced significantly by NDRG1 in the two cancer cell lines relative to control cells (Fig. 3B). These data suggested that HIF1 $\alpha$ could be a downstream target of NDRG1 in current circumstance and inhibited by NDRG1 in an Akt/mTOR signaling-mediated manner.

Considering the marked effect of NDRG1 on HIF1 $\alpha$ expression in pancreatic cancer cells, further investigations were conducted to examine this effect in human PDAC specimens by immunohistochemistry (Fig. 3C). Similarly, there was a negative correlation between NDRG1 and HIF1 $\alpha$ expression in PDAC, which is consistent with our in vitro data using MIA PaCa-2 and PANC-1 cells demonstrating the inhibitory effect of NDRG1 on HIF1 $\alpha$ expression in PDAC (Fig. 3C).

NDRG1 inhibits glucose metabolism and promotes mitochondrial respiration in pancreatic cancer cells. The ECAR is an important measurement of glucose metabolism and 
A

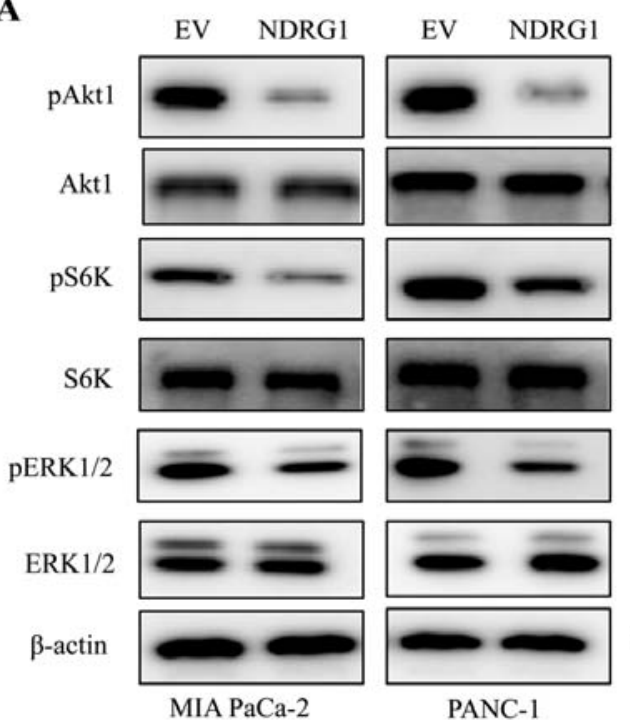

B

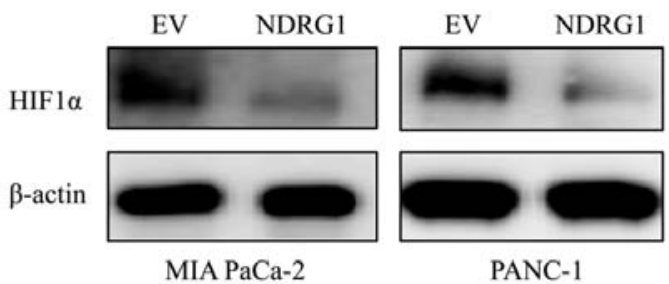

C
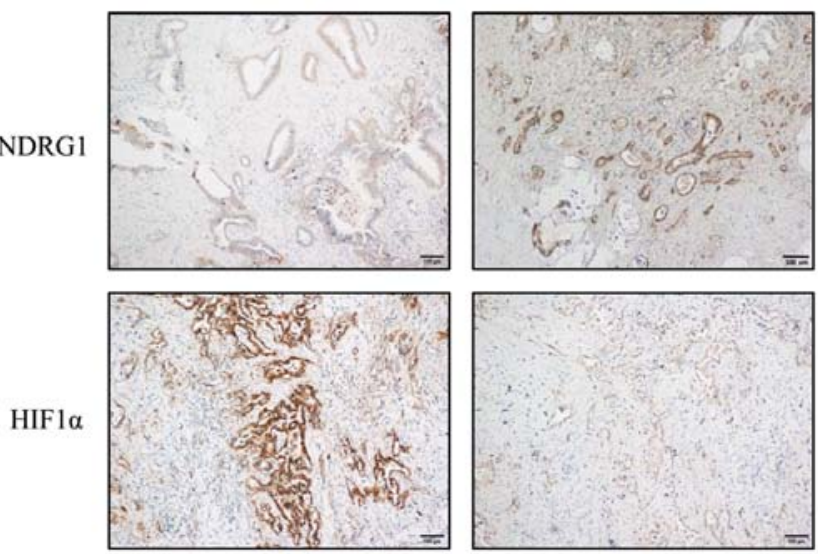

Figure 3. NDRG1 decreases HIF1 $\alpha$ expression through MAPK and Akt/mTOR signaling. (A) Immunoblotting showed that NDRG1 decreased phosphorylated ERK1/2, Akt1 and S6K level other than total ERK1/2, Akt1 and S6K. Blots are representative of 3-5 experiments. (B) Immunoblotting suggested that HIF1a protein level was reduced upon NDRG1 overexpression. (C) Immunohistochemistry was conducted to examine change of HIF1 $\alpha$ expression with different NDRG1 abundance in human pancreatic cancer specimens.
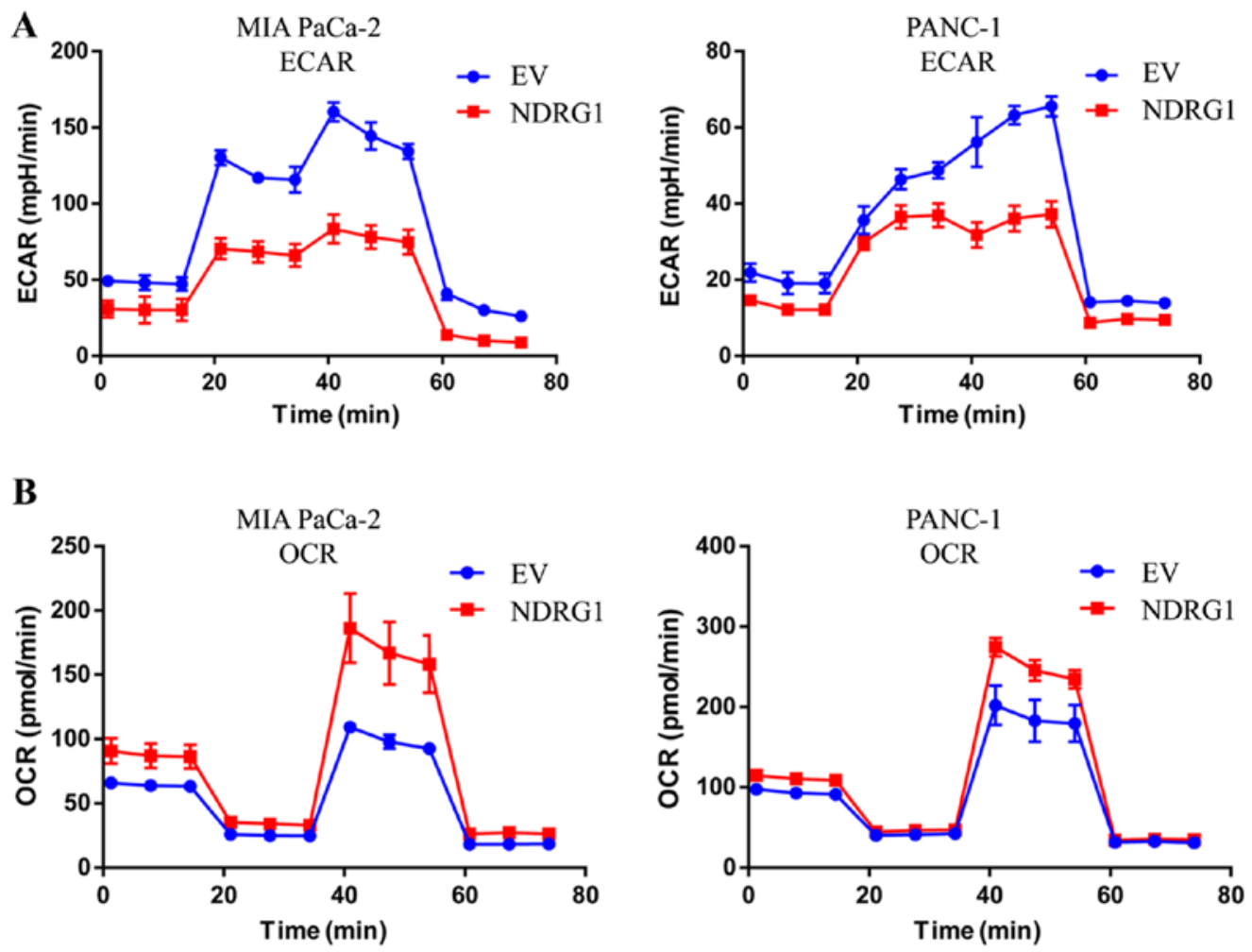

Figure 4. NDRG1 inhibits glucose metabolism while promotes mitochondrial respiration in pancreatic cancer cells. ECAR and OCR were performed (see Materials and methods) to determine the effect of NDRG1 on glucose metabolism and mitochondrial respiration of pancreatic cancer cells. (A) NDRG1 expression decreased the ECAR as expected in both cell lines. (B) OCR experiment demonstrated NDRG1 positive mitochondrial respiration in pancreatic cancer cells. 
A
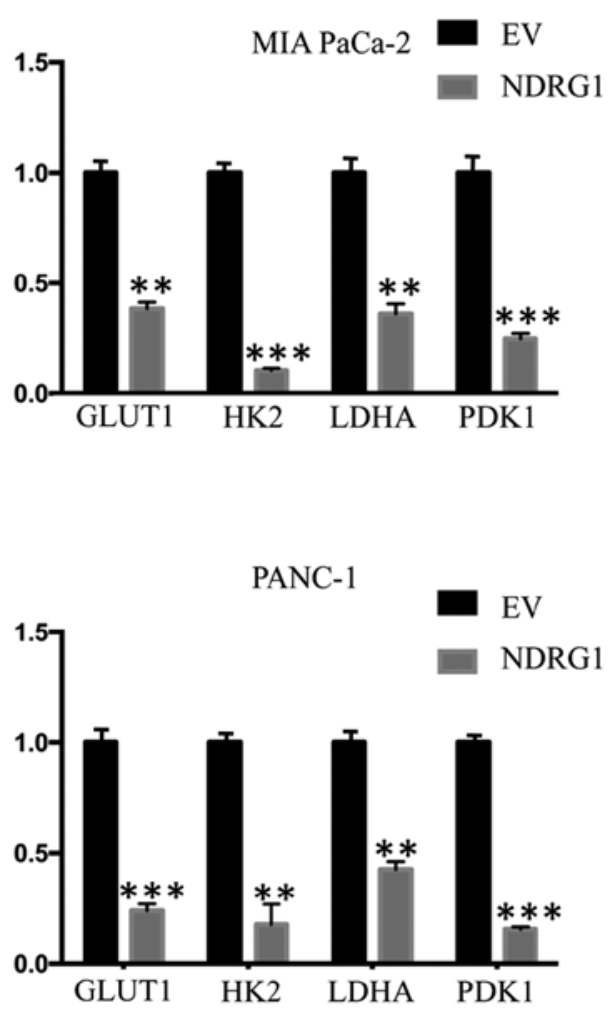

B

GLUT1

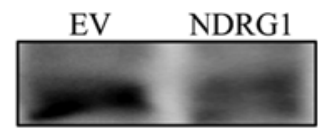

HK2

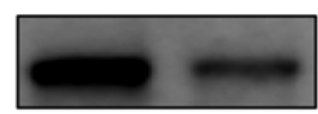

LDHA

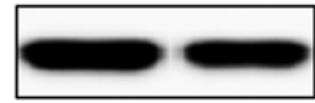

PDK1

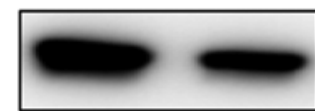

$\beta$-actin

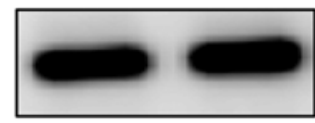

MIA PaCa-2

C

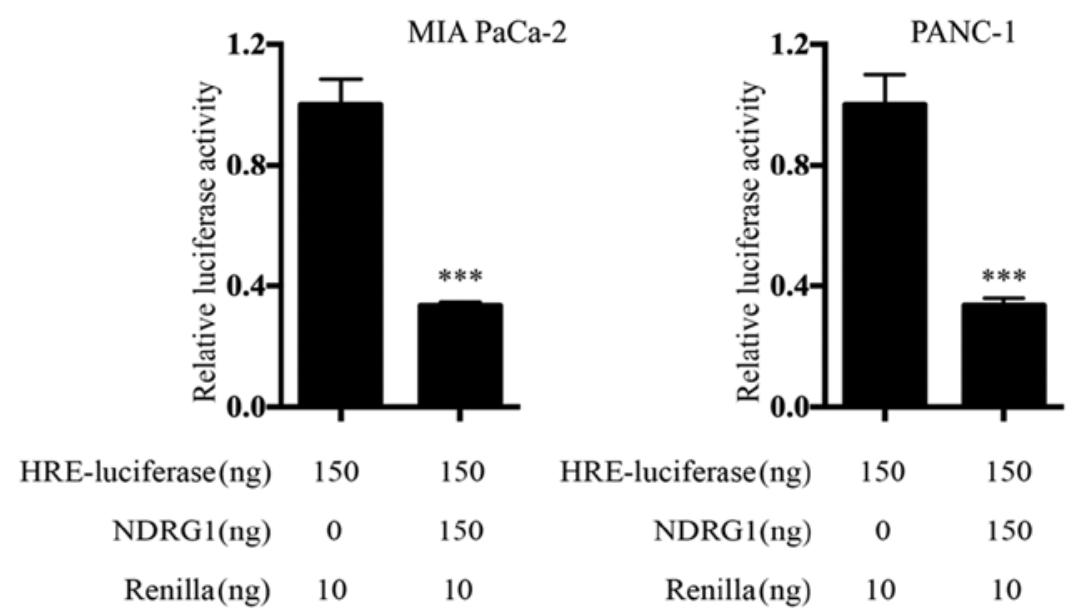

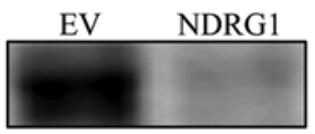
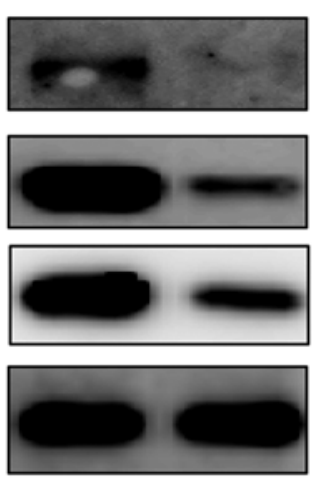

PANC-1

Figure 5. NDRG1 downregulates transcription and expression of glucose metabolism related enzymes. (A) qRT-PCR was explored to investigate whether mRNA of GLUT1, HK2, LDHA and PDK1 were affected by NDRG1. Data represent the means \pm SD from three independent experiments. (B) Immunoblotting presented that GLUT1, HK2, LDHA and PDK1 protein level was deceased by NDRG1 overexpression. (C) HRE reporter assay was used to assess HIF1a activity. Data represent the means \pm SD from three independent experiments. ${ }^{*} \mathrm{P}<0.05,{ }^{* * *} \mathrm{P}<0.01,{ }^{* * * *} \mathrm{P}<0.001$, relative to control cells.

reflects the lactic acid-induced acidification of the medium surrounding cancer cells. Considering the inhibitory effect of NDRG1 on pancreatic cancer cell growth, further studies were conducted to examine whether NDRG1 could modulate glycolysis metabolism of these pancreatic cancer cells, which control the energy feed for cell growth. Noteworthy, in both MIA PaCa-2 and PANC-1 cells NDRG1 expression decreased the ECAR as expected (Fig. 4A), especially from 20 min to $60 \mathrm{~min}$, which imply that it plays a suppressive role in lactic acid formed during glycolysis. Cellular oxygen consumption reflects mitochondrial respiration and can be measured by the OCR. To further investigate this novel finding, the above studies were complemented by OCR experiment to assess the effect of NDRG1 on the 'metabolism switch'. Conversely, OCR experiment demonstrated that when MIA PaCa-2 and PANC-1 cells overexpress NDRG1, they exhibited higher OCRs comparing to control cells (Fig. 4B), indicating that NDRG1 is a positive regulator of basal mitochondrial respiration in pancreatic cancer cells.

NDRG1 downregulates transcription and expression of glucose metabolism related enzymes. The studies above indicated that
NDRG1 suppress pancreatic cancer cells metabolic level of glucose, while enhanced the mitochondrial function. To further elucidate the underlying mechanism of NDRG1 function, we conducted qRT-PCR to examine whether several key enzymes of glucose metabolism, including GLUT1, HK2, LDHA and PDK1, were affected by NDRG1 at mRNA level. For both MIA PaCa-2 and PANC-1 cells, we observed consisted results that the mRNA level of GLUT1, HK2, LDHA and PDK1 was significantly decreased upon NDRG1 expression, relative to the vector control $(\mathrm{P}<0.05)$ (Fig. 5A). Considering our data above showing that NDRG1 significantly decreased GLUT1, HK2, LDHA and PDK1 mRNA in the two cell lines, further studies were performed to examine whether this effect was occurring at the protein level. Therefore, we applied immunoblotting to verify protein levels of GLUT1, HK2, LDHA and PDK1 in response to NDRG1 in both cell-types. Similarly, in comparison to the control cells, there was a marked reduction of GLUT1, HK2, LDHA and PDK1 protein in the presence of NDRG1 overexpression in both cell lines (Fig. 5B). In summary, these results suggest that the effect of NDRG1 on these key enzymes is occurring at both transcription and post-transcription levels. 


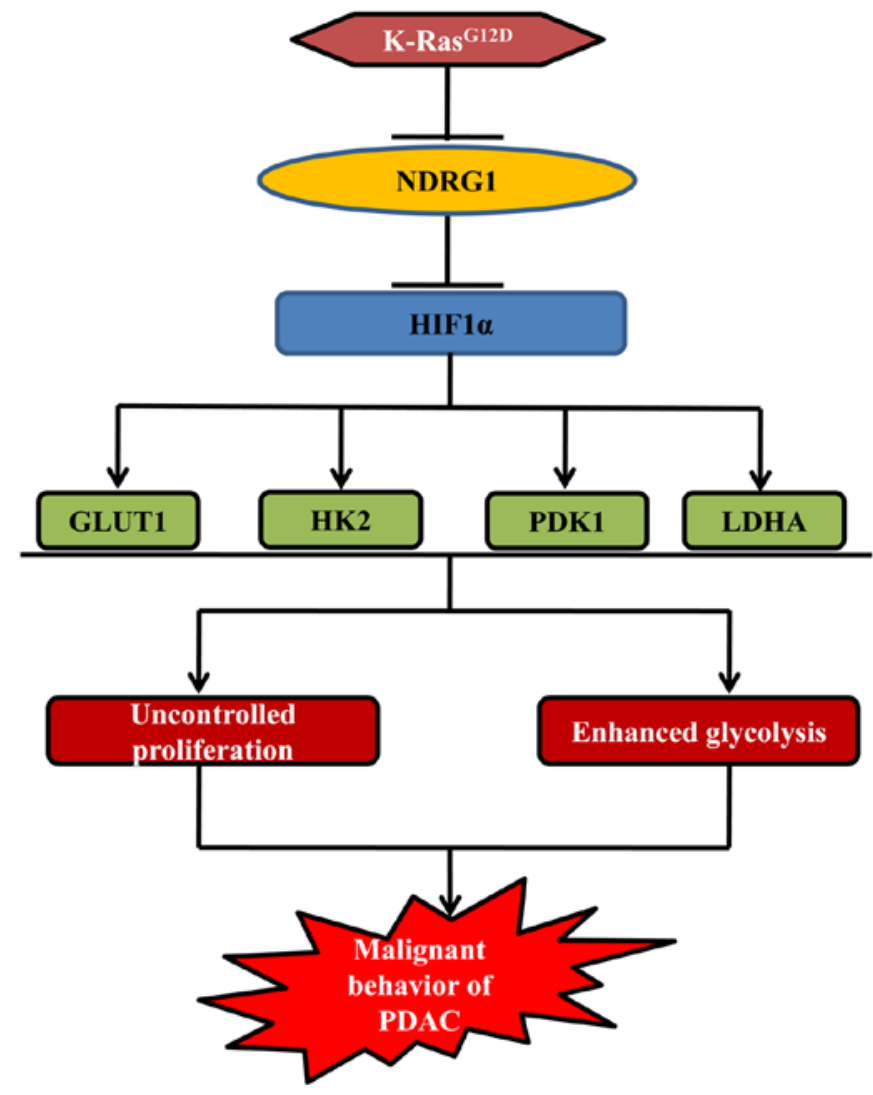

Figure 6. Schematic illustration summarizing the inhibitory effect of NDRG1 on glycolysis in pancreatic cancer as suggested in this investigation. K-Ras plays a vital role in modulating NDRG1 protein level in PDAC cancer cells in vitro, which is mediated through ERK signaling. Noteworthy, K-Ras downstream Akt/mTOR signaling is inhibited upon NDRG1 expression, resulting in decease of HIF1 $\alpha$ level and activity. Moreover, NDRG1 has a unique role in modulating cancer metabolism of PDAC. The mechanism of inhibiting glycolysis of PDAC involves the NDRG1-mediated downregulation of several glycolysis key enzymes including GLUT1, HK2, LDHA and PDK1. Additionally, NDRG1 is shown to suppress the activity of HIF1 $\alpha$, which may be responsible for inhibition of glycolysis enzymes.

To decipher the inhibitory effect of NDRG1 on metabolism of pancreatic cancer cells which typically grow within hypoxic conditions, we further performed investigation using HRE reporter assay to assess the role of NDRG1 on HIF1 $\alpha$ activity. Both cell lines were transfected with the reporter plasmid. For MIA PaCa-2 cells, NDRG1 overexpression led to significant decrease of HIF1 $\alpha$ transcriptional activity relative to control cells $(\mathrm{P}<0.05)$, as reflected by HRE-luciferase reporter assay. A similar effect was also observed for PANC-1 cells (Fig. 5C). These results showed that NDRG1 prevented activity of HIF1 $\alpha$ which resulted in the decrease of the four glycolysis enzymes. Collectively, NDRG1 overexpression remarkably reduced mRNA and protein level of GLUT1, HK2, LDHA and PDK1, which is achieved by blocking activity of HIF1 $\alpha$.

\section{Discussion}

A series of evidenc has supported the role of NDRG1 in inhibiting cancer growth, development and metastasis (11,18-21). However, complete understanding of its mechanism of action remains an important research goal. This current investigation demonstrates that mutant/activate K-Ras reduces NDRG1 protein via ERK-mediated signaling (Fig. 1). Moreover, we discovered a unique role of NDRG1 in modulating PDAC cancer cell glycolysis, resulting in inhibited cell growth (Fig. 2), which is achieved through NDRG1-induced downregulation of HIF1 $\alpha$ protein levels and activity (Figs. 3B and 5C). This effect is further confirmed by immunohistochemical analysis using human PDAC specimens (Fig. 3C). Furthermore, many glycolysis related-enzymes are found to be reduced at mRNA and protein levels upon NDRG1 overexpression (Fig. 5A and B) and, hence, ECAR as well as OCR is concurrently changed (Fig. 4A and B). Significantly, this is the first time that this potent metastasis suppressor has been shown to take part in metabolic regulation of PDAC glycolysis (Fig. 6).

An emerging theme in cancer biology is that many of the oncogenic mutations which result in tumorigenesis drive the altered metabolism of cancer cells $(2,5,7)$. Cancer cells are addicted to use glycolysis other than mitochondrial oxidative phosphorylation for glucose-dependent ATP production, no matter whether ample oxygen is provided or not, to fuel mitochondrial respiration, a phenomenon known as the 'Warburg effect' (4). Not surprisingly, given its central role in the pathogenesis of PDAC, oncogenic K-Ras has been recently shown to have a key role in multiple aspects of PDAC metabolism $(22,23)$. Of note, it seems that a number of well-known tumor suppressors play critical roles in modulating the 'metabolic transformation' to weaken the 'Warburg effect' (24). It is believed that oncogene and tumor suppressor networks influence the metabolic shift in cancer. Hence, the potent tumor/metastasis suppressor NDRG1, which had been demonstrated to negatively affect cancer growth of PDAC (10), was investigated here in K-Ras network to understand the precise role in PDAC. Our data showed that mutant/activated K-Ras decreased NDRG1 protein level (Fig. 1A and B). However, when ERK1, the effector downstream of K-Ras, was abrogated by siRNA or specific inhibitor U0126, the inhibitory effect of K-Ras on NDRG1 was reversed indicating that K-Ras inhibits NDRG1 through ERK-mediated signaling.

The K-Ras oncogene has been shown to promote glycolysis so as to drive uncontrolled proliferation and enhance survival of cancer cells (5). For instance, the glucose transporter GLUT1 is transcriptionally upregulated in response to K-Ras mutations, leading to elevated glucose uptake and glycolysis $(5,25)$. In addition to the above mentioned case, glutamine consumption, anomalous pentose phosphate pathway (PPP) and autophagy is also enhanced downstream of K-Ras in different and complementary ways $(5,26)$, resulting in 'metabolic addiction' of pancreatic tumor cells. Cell proliferation assay and colony formation assay results suggested that cell growth of pancreatic cancer cells was significantly suppressed by NDRG1 (Fig. 2), which is in good agreement with previous studies $(10,27)$. The ERK1 and Akt/mTOR signaling pathway are direct downstream effectors of Ras, contributing to Ras-driven oncogenesis (15). However, NDRG1 abrogate cancer cell growth of PDAC cancer cells $(10,27)$. Noteworthy, our results demonstrated overexpression of NDRG1 led to significant reduction of phosphorylated Akt1, S6K and ERK1 protein, but had no change on total Akt1, S6K and ERK1 protein level. These data prove that NDRG1 has inhibitory effect on MAPK and Akt/mTOR signaling in K-Ras network, and the interplay of NDRG1 and ERK1 is context-dependent. 
As mentioned above, tissue within PDAC is highly hypoxic. Not surprisingly, this hypoxic microenvironment leads to its complex biology and in particular its cellular metabolism (28). HIF1 $\alpha$ is an important regulator of cellular oxygen homeostasis, and is overexpressed in pancreatic cancer and associated with poor prognosis (6). Moreover, it is suggested that HIF1 $\alpha$ promotes PDAC progression by upregulating VEGF, which is a key inducer of angiogenesis (29). Intriguingly, NDRG1 expression is associated with a higher differentiation status of the cells and correlated with a better prognosis as well as longer survival in patients with pancreatic cancer (10). NDRG1 was demonstrated to affect the angiogenic on or off switch of cancer stroma in PDAC through attenuation of NF- $\mathrm{KB}$ signaling pathway $(27,30)$. Importantly, Akt/mTOR signaling has been shown to stimulate translation of HIF1 $\alpha$ mRNA and elevate HIF1 $\alpha$ protein level (16). The present study showed that NDRG1 overexpression decreased both protein level and activity of HIF1 $\alpha$ (Fig. 3B). Immunohistochemical analysis of PDAC specimens further provided supports that high NDRG1 was correlated with reduced HIF1 $\alpha$ staining (Fig. 3C). Collectively, these studies suggest that abundant NDRG1 protein acts as suppressor of HIF1 $\alpha$ in PDAC. However, more investigations are needed to elucidate the underlying mechanisms.

One of the consequences of elevated HIF1 $\alpha$ is the metabolic rewiring allowing a cell to subsist in low-oxygen environment. The transcription factor HIF1 $\alpha$ has been implicated in regulating many of the genes that are responsible for the metabolic difference. Most part of glycolysis related enzymes are directly or indirectly regulated by HIF1 $\alpha$, in the way that HIF1 $\alpha$ coordinately modulate the entire process (31). In PDAC, HIF1 $\alpha$ has been shown to mediate metabolic changes downstream of MUC1 in vitro and in vivo (32). HIF1 $\alpha$ stimulates glycolytic energy production by trans-activating genes involved in GLUT1 (33), inducing LDHA and HK2 expression to catalyze lactate conversion and phosphorylate glucose $(34,35)$. Using qRT-PCR and western, we found that NDRG1 reduced GLUT1, HK2, LDHA and PDK1 mRNA and protein level in both cell lines (Fig. 5A and B), which is accompanied by HIF $1 \alpha$ transcriptional activity reduction (Fig. 5C).

In conclusion, the investigation herein highlights a novel facet of NDRG1 in K-Ras network (Fig. 6). NDRG1 inhibits PDAC cancer cell growth via modulating glycolytic metabolism of PDAC (Fig. 6), which is achieved through suppression of several glycolysis associated key enzymes (namely GLUT1, HK2, LDHA and PDK1) at both mRNA and protein levels, leading to decreased ECAR and improved mitochondrial respiration (Fig. 6). Moreover, NDRG1-mediated reduction of GLUT1, HK2, LDHA and PDK1 is downstream of HIF1 $\alpha$ which was proved to be inhibited by NDRG1 overexpression in PDAC cancer cell lines as well as specimens (Fig. 6). Therefore, NDRG1 inhibits HIF1 $\alpha$ and its downstream key enzymes of glycolysis to exert its notable anti-metabolic switch activity in PDAC.

\section{Acknowledgements}

This study was supported by the National Natural Science Foundation of China (nos. 81372651, 81502031 and 81602058), Science and Technology Commission of Shanghai Municipality (13DZ1942802 and 14ZR1407700) and Youth Program of Shanghai Municipal Health Bureau (20154Y0090).

\section{References}

1. Kamisawa T, Wood LD, Itoi T and Takaori K: Pancreatic cancer. Lancet 388: 73-85, 2016.

2. Le A, Rajeshkumar NV, Maitra A and Dang CV: Conceptual framework for cutting the pancreatic cancer fuel supply. Clin Cancer Res 18: 4285-4290, 2012.

3. Sousa CM and Kimmelman AC: The complex landscape of pancreatic cancer metabolism. Carcinogenesis 35: 1441-1450, 2014.

4. Cantor JR and Sabatini DM: Cancer cell metabolism: One hallmark, many faces. Cancer Discov 2: 881-898, 2012.

5. Ying H, Kimmelman AC, Lyssiotis CA, Hua S, Chu GC, Fletcher-Sananikone E, Locasale JW, Son J, Zhang H, Coloff JL, et al: Oncogenic Kras maintains pancreatic tumors through regulation of anabolic glucose metabolism. Cell 149: 656-670, 2012.

6. Meijer TW, Kaanders JH, Span PN and Bussink J: Targeting hypoxia, HIF-1, and tumor glucose metabolism to improve radiotherapy efficacy. Clin Cancer Res 18: 5585-5594, 2012.

7. Li B and Simon MC: Molecular Pathways: Targeting MYC-induced metabolic reprogramming and oncogenic stress in cancer. Clin Cancer Res 19: 5835-5841, 2013.

8. Miller DM, Thomas SD, Islam A, Muench D and Sedoris K: c-Myc and cancer metabolism. Clin Cancer Res 18: 5546-5553, 2012.

9. Smith SC and Theodorescu D: Learning therapeutic lessons from metastasis suppressor proteins. Nat Rev Cancer 9: 253-264, 2009.

10. Angst E, Dawson DW, Stroka D, Gloor B, Park J, Candinas D, Reber HA, Hines OJ and Eibl G: N-myc downstream regulated gene-1 expression correlates with reduced pancreatic cancer growth and increased apoptosis in vitro and in vivo. Surgery 149: 614-624, 2011.

11. Liu W, Yue F, Zheng M, Merlot A, Bae DH, Huang M, Lane D, Jansson P, Lui GY, Richardson V, et al: The proto-oncogene $\mathrm{c}$-Src and its downstream signaling pathways are inhibited by the metastasis suppressor, NDRG1. Oncotarget 6: 8851-8874, 2015.

12. Jain S, Wang $X$, Chang CC, Ibarra-Drendall C, Wang $H$, Zhang Q, Brady SW, Li P, Zhao H, Dobbs J, et al: Src inhibition blocks c-Myc translation and glucose metabolism to prevent the development of breast cancer. Cancer Res 75: 4863-4875, 2015.

13. Ji S, Qin Y, Liang C, Huang R, Shi S, Liu J, Jin K, Liang D, $\mathrm{Xu}$ W, Zhang B, et al: FBW7 (F-box and WD repeat domaincontaining 7) negatively regulates glucose metabolism by targeting the c-Myc/TXNIP (thioredoxin-binding protein) axis in pancreatic cancer. Clin Cancer Res 22: 3950-3960, 2016.

14. Emerling BM, Weinberg F, Liu JL, Mak TW and Chandel NS: PTEN regulates p300-dependent hypoxia-inducible factor 1 transcriptional activity through Forkhead transcription factor $3 \mathrm{a}$ (FOXO3a). Proc Natl Acad Sci USA 105: 2622-2627, 2008.

15. Kennedy AL, Adams PD and Morton JP: Ras, PI3K/Akt and senescence: Paradoxes provide clues for pancreatic cancer therapy. Small GTPases 2: 264-267, 2011.

16. Harada H, Itasaka S, Kizaka-Kondoh S, Shibuya K, Morinibu A, Shinomiya K and Hiraoka M: The Akt/mTOR pathway assures the synthesis of HIF-1alpha protein in a glucose- and reoxygenationdependent manner in irradiated tumors. J Biol Chem 284: 5332-5342, 2009.

17. Denko NC: Hypoxia, HIF1 and glucose metabolism in the solid tumour. Nat Rev Cancer 8: 705-713, 2008.

18. Kurdistani SK, Arizti P, Reimer CL, Sugrue MM, Aaronson SA and Lee SW: Inhibition of tumor cell growth by RTP/rit42 and its responsiveness to p53 and DNA damage. Cancer Res 58: 4439-4444, 1998.

19. Guan RJ, Ford HL, Fu Y, Li Y, Shaw LM and Pardee AB: Drg-1 as a differentiation-related, putative metastatic suppressor gene in human colon cancer. Cancer Res 60: 749-755, 2000.

20. Bandyopadhyay S, Pai SK, Gross SC, Hirota S, Hosobe S, Miura K, Saito K, Commes T, Hayashi S, Watabe M, et al: The Drg-1 gene suppresses tumor metastasis in prostate cancer. Cancer Res 63: 1731-1736, 2003. 
21. Liu W, Xing F, Iiizumi-Gairani M, Okuda H, Watabe M, Pai SK, Pandey PR, Hirota S, Kobayashi A, Mo YY, et al: N-myc downstream regulated gene 1 modulates Wnt- $\beta$-catenin signalling and pleiotropically suppresses metastasis. EMBO Mol Med 4: 93-108, 2012.

22. Mann KM, Ying H, Juan J, Jenkins NA and Copeland NG: KRAS-related proteins in pancreatic cancer. Pharmacol Ther 168: 29-42, 2016.

23. Logsdon CD and Lu W: The significance of Ras activity in pancreatic cancer initiation. Int J Biol Sci 12: 338-346, 2016.

24. Jones RG and Thompson CB: Tumor suppressors and cell metabolism: A recipe for cancer growth. Genes Dev 23: 537-548, 2009.

25. Yun J, Rago C, Cheong I, Pagliarini R, Angenendt $\mathrm{P}$ Rajagopalan H, Schmidt K, Willson JK, Markowitz S, Zhou S, et al: Glucose deprivation contributes to the development of KRAS pathway mutations in tumor cells. Science 325: 1555-1559, 2009.

26. Guo JY, Chen HY, Mathew R, Fan J, Strohecker AM, KarsliUzunbas G, Kamphorst JJ, Chen G, Lemons JM, Karantza V, et al: Activated Ras requires autophagy to maintain oxidative metabolism and tumorigenesis. Genes Dev 25: 460-470, 2011.

27. Hosoi F, Izumi H, Kawahara A, Murakami Y, Kinoshita $\mathrm{H}$, Kage M, Nishio K, Kohno K, Kuwano M and Ono M: N-myc downstream regulated gene 1/Cap43 suppresses tumor growth and angiogenesis of pancreatic cancer through attenuation of inhibitor of kappaB kinase beta expression. Cancer Res 69: 4983-4991, 2009.

28. Guillaumond F, Leca J, Olivares O, Lavaut MN, Vidal N, Berthezène $\mathrm{P}$, Dusetti NJ, Loncle $\mathrm{C}$, Calvo $\mathrm{E}$, Turrini $\mathrm{O}$, et al: Strengthened glycolysis under hypoxia supports tumor symbiosis and hexosamine biosynthesis in pancreatic adenocarcinoma. Proc Natl Acad Sci USA 110: 3919-3924, 2013.
29. Sun HC, Qiu ZJ, Liu J, Sun J, Jiang T, Huang KJ, Yao M and Huang C: Expression of hypoxia-inducible factor- $1 \alpha$ and associated proteins in pancreatic ductal adenocarcinoma and their impact on prognosis. Int J Oncol 30: 1359-1367, 2007.

30. Maruyama Y, Ono M, Kawahara A, Yokoyama T, Basaki Y, Kage M, Aoyagi S, Kinoshita H and Kuwano M: Tumor growth suppression in pancreatic cancer by a putative metastasis suppressor gene Cap43/NDRG1/Drg-1 through modulation of angiogenesis. Cancer Res 66: 6233-6242, 2006.

31. Iyer NV, Kotch LE, Agani F, Leung SW, Laughner E, Wenger RH, Gassmann M, Gearhart JD, Lawler AM, Yu AY, et al: Cellular and developmental control of $\mathrm{O} 2$ homeostasis by hypoxiainducible factor 1 alpha. Genes Dev 12: 149-162, 1998.

32. Chaika NV, Gebregiworgis T, Lewallen ME, Purohit V, Radhakrishnan P, Liu X, Zhang B, Mehla K, Brown RB, Caffrey T, et al: MUC1 mucin stabilizes and activates hypoxiainducible factor 1 alpha to regulate metabolism in pancreatic cancer. Proc Natl Acad Sci USA 109: 13787-13792, 2012.

33. Chen C, Pore N, Behrooz A, Ismail-Beigi F and Maity A: Regulation of glut 1 mRNA by hypoxia-inducible factor- 1 . Interaction between H-ras and hypoxia. J Biol Chem 276: 9519-9525, 2001.

34. Firth JD, Ebert BL and Ratcliffe PJ: Hypoxic regulation of lactate dehydrogenase A. Interaction between hypoxia-inducible factor 1 and cAMP response elements. J Biol Chem 270: 21021-21027, 1995.

35. Mathupala SP, Rempel A and Pedersen PL: Glucose catabolism in cancer cells: Identification and characterization of a marked activation response of the type II hexokinase gene to hypoxic conditions. J Biol Chem 276: 43407-43412, 2001. 